\section{Fatty plaque link to inflammation}

A major constituent of the fatty arterial plaques seen in heart disease may dampen the inflammation that drives the disease. A cholesterolprecursor molecule may be a mediator of this suppression.

Atherosclerosis progresses as cholesterol-filled immune cells called macrophage foam cells accumulate in arterial walls. Christopher Glass at the University of California, San Diego, and his colleagues found that in mice fed a high-fat, high-cholesterol diet, these cells were linked to suppression of inflammation. Within the foam cells, desmosterol, an intermediate in the cholesterol biosynthesis pathway, accumulated to significantly higher levels than did other intermediates. Moreover, desmosterol was also abundant in human atherosclerotic lesions. In the mice, desmosterol seemed to suppress genes that promote inflammation during foam-cell formation.

Synthetic formulations of desmosterol might intervene in cardiovascular disease, the authors suggest.

Cell 151, 138-152 (2012)

ASTRONOMY

\section{Homing in on a black hole's jets}

Astronomers have for the first time located the launch site of a giant, high-speed jet of charged particles believed to originate from a supermassive black hole.

Black holes suck up large amounts of gas and dust, which swirl around the black hole and are thought to feed these jets (simulated image pictured). Sheperd Doeleman of the Massachusetts Institute of Technology's Haystack Observatory in Westford and his colleagues linked four radio dishes in California, Arizona and Hawaii to make a single large telescope. Using this, they examined the jets' source region, which lies just outside the 6.2-billion-solarmass black hole at the centre of the galaxy M87.

The launch site was small, suggesting two key properties about the gravitational giant: the black hole spins, and it feeds on a surrounding disk of material that orbits it in the same direction as its spin.

Science http://dx.doi.

org/10.1126/science.1224768

(2012)

For a longer story on this research, see go.nature.com/tqrwgb

\section{CELL BIOLOGY}

\section{A way to catch dividing cells}

A genetic tool can help biologists to pinpoint rare, replicating cells in tissue samples from adult mice.

Finding and studying dividing cells is important in learning about growth, healing and other key processes, but researchers have been hindered by the difficulty of isolating these cells alive. To overcome this problem, Amir Eden at the Hebrew University of Jerusalem and his colleagues created a mouse strain in which the gene for a fluorescent protein was fused to a gene that is active when cells divide. After dissecting livers from these mice, the researchers could sort dividing from nondividing cells and compare their gene expression. Genes

COMMUNITY CHOICE

The most viewed papers in science

\title{
ELECTRONICS
}

\section{Bendable battery yields flexible LED}

HIGHLY READ
on pubs.acs.org
in August

Thanks to an energy-dense flexible lithium-ion battery, researchers have built a thin, bendable light source using an organic light-emitting diode (LED).

The device could one day be incorporated into rollable or implantable electronics.

Lithium-ion batteries are among the best candidates for flexible power sources, but their electrodes could previously be made with only a few, low-performing materials. Now, Keon Jae Lee at the Korea Advanced Institute of Science and Technology in Daejeon and his colleagues have created a bendable battery by using existing methods to apply lithium-based electrodes onto a brittle mica surface at high temperature and then peel off the mica substrate. They then used a technique that they had devised to transfer the battery onto a flexible polymer. The method allows the incorporation into a flexible battery of almost all the high-performance materials that are used in rigid batteries.

Nano Lett. 12, 4810-4816 (2012)

for liver specialization, or differentiation, were less active in replicating cells.

This fluorescent marker could help scientists to isolate and study dividing cells in multiple tissues and biological assays.

Dev. Cell http://

dx.doi.org/10.1016/j.

devcel.2012.08.009 (2012)

\section{NEUROSCIENCE}

\section{Zebrafish find light without eyes}

Eyeless zebrafish larvae may still find their way out of darkness by the activation of light-sensitive neurons deep inside the brain.

Harold Burgess at the National Institute of Child Health and Human Development in Bethesda, Maryland, and his team found that the transparent zebrafish larvae (Danio rerio; pictured, with certain brain cells in green) swim gradually towards the illuminated areas of their tank, even after their eyes have been removed. This behaviour suggests the

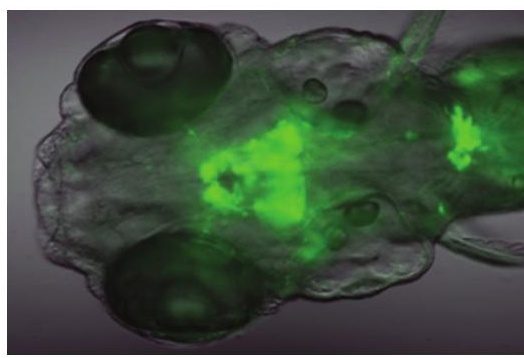

presence of light-responsive neurons outside the conventional visual organs. Engineered eyeless fish that express less Opn $4 a-a$ light-sensitive molecule in the brain - responded poorly to light, whereas fish that produced more Opn4a performed better.

The researchers conclude that neurons expressing Opn4a in the preoptic area of the brain may support simple, and perhaps primordial, light-seeking behaviours.

Curr. Biol. http://dx.doi. org/10.1016/j.cub.2012.08.016 (2012)

\section{$\rightarrow$ NATURE.COM}

For the latest research published by Naturevisit:

www.nature.com/latestresearch 